\title{
Medical Image Compression Based on Combining Region Growing and Wavelet Transform
}

\author{
EInomery Allam Zanaty ${ }^{1}$, Sherif Mostafa Ibrahim ${ }^{2}$ \\ ${ }^{1}$ Faculty of Computers \& Information, Sohag University, Sohag, Egypt \\ ${ }^{2}$ Mathimatics \& Computer Science Department, Faculty of Science, South Valley University, Qena, Egypt
}

Email address:

zanaty22@yahoo.com (E. A. Zanaty)

\section{To cite this article:}

Elnomery Allam Zanaty, Sherif Mostafa Ibrahim. Medical Image Compression Based on Combining Region Growing and Wavelet Transform. International Journal of Medical Imaging. Vol. 7, No. 3, 2019, pp. 57-65. doi: 10.11648/j.ijmi.20190703.11

Received: August 20,2019; Accepted: September 17, 2019; Published: September 27, 2019

\begin{abstract}
Medical data grows very fast and hence medical institutions need to store high volume of data about their patients. Medical images are one of the most important data types about patients. As a result, hospitals have a high volume of images that require a huge storage space and transmission bandwidth to store these images. Most of the time transmission bandwidth is not sufficient to storing and transmit all the image data with the required efficiency. Image compression is the process of encoding information using fewer bits than an un-encoded representation using specific encoding schemes. Compression is useful because it helps to reduce the consumption of expensive resources, such as storage space or transmission bandwidth (computing). In this paper, a medical image compression technique based on combining region growing and wavelets algorithms was introduced. A region growing algorithm is used to simply partitioning the image into two parts foreground and background depending on the intensity values. Then, wavelets methods applied on foreground regions including important regions. These regions are compressed lossless to keep the appearance of the image as intact while making the simplifications and the other region is lossy compressed to reduce the file size, leading to that the overall compression ratio gets better and the reconstructed image seems like the original one. To prove the capability of the proposed algorithm, different four image structures from X-Ray, Computed tomography CT and Magnetic resonance imaging MRI types are tested.
\end{abstract}

Keywords: Wavelets Transform, Region Growing, SPIHT, Daubechies, Biorthogonal

\section{Introduction}

The need for compressing medical image is increasing daily cause every hospital have to keep archive to store the patient history including their different types of medical images. Many researches strive and achieve good results. Julius introduced a way of compressing medical images by using Discrete Haar wavelet transform (DWT) for improving compression efficiencies for monochrome and color images [1]. Rema had proposed a new means of reordering spatial orientation tree of SPIHT. Reordering ensured that SPIHT algorithm codes more important information in the initial bits. List of insignificant pixels and sets are initialized with less number of coefficients compared to conventional SPIHT for monochrome images [2]. Ramakrishnan and Sriraam has developed a new image compression technique in the field on medical images called internet transmission of DICOM (Digital Imaging and Communications in Medicine) with effective bandwidth utilization. For the progressive transmission of DICOM images a wavelet based encoder called SPIHT (set partitioning in hierarchical trees) has been used [3]. Chen had designed \& implemented a new medical image compression technique based on subband decomposition called DCT and modified SPIHT (set partitioning in hierarchical trees) data organization. Discrete Cosine Transform (DCT) was used for the subband decomposition. The detailed features of an image were stored in the translation function. In this method, high-frequency sub bands are used in good number for reduction of the redundancy by using the algorithm with modified SPIHT. Experimental results showed that the quality of the reconstructed medical image has been increased by the peak signal-to-noise ratio (PSNR) value [4]. Sharma introduced multilevel three-dimensional discrete wavelet change (3DDWT) modules for digital image compression. The proposed architecture depends on lifting scheme 
methodology; the main flavour of the design is minimized storage requirement and memory referencing [5]. Maye designed coherent 13/7 three dimensional discrete wavelets transform for image compression in VLSI architectures to overcome the problem of convention methods [6].

Sridhar mainly focused upon the comparison and analysis of image compression metrics derived by the application of different wavelets like Haar, Daubechies, Coiflet, Symlet and Biorthogonal wavelets on various selected bench mark grey scale and color images of varying content and details [7].

Vasanth et al. [8] merged discrete wavelet transform together with oppositional based grey wolf optimization method to enhance the coefficient for Haar wavelet values then compression strategy module based encryption is done [8].

Basma introduced two different techniques of mammogram compression. The algorithm used includes two main steps [9]. A pre-processing step is applied to enhance the image, and then a compression algorithm is applied to the enhanced image, they found that Comparable results were obtained when applying image enhancement before the compression process.

In this paper, a compression technique for medical image introduced to create a compact data representation for storage and transmission purposes. The proposed technique combines region growing and wavelets algorithms. It gives the advantages of low complexity, better results, also reduce the adders and multipliers, Throughput increased by three times, and latency rate gets reduced. Objective fidelity measures evaluated like PSNR and MSE relative merits of different Wavelet transform techniques.

The paper is organized as follows: In section 2, region growing segmentation algorithm described. Wavelets algorithms presented in Section 3. We present the proposed algorithms in Section 4. Section 5 shows the performance evaluation metrics. In section 6 , experimental results analysis discussed. Conclusion \& future work is described in section 7.

\section{Region Growing (RG)}

Image segmentation approaches commonly based on one of two fundamental properties of intensity values. In discontinuity based techniques image is portioned by sudden changes in intensity values whereas similarity based technique partitions an image by grouping together connected pixels in the region which fulfils predefined resemblance criteria. Simply The region growing (RG) algorithm starts with a set of seed points, each corresponding to an individual region compared to their neighbours based on a similarity criterion. The neighbours of a pixel are computed with its 8-connectivity. The simplest similarity criteria generally used is obtained by calculating the difference between the intensity value of the image pixel and the corresponding region mean. If the difference is less than a specified threshold, then the pixel belongs to that region and is subsequently labelled. Otherwise the pixel is not labelled and skipped. Zanaty and Abdel-hafiz [10] performed a deep study for the various 8 classical segmentation techniques and region growing give overall better results than many other algorithms [11-12].

\section{Wavelets}

Daubechies wavelets: are defined [13] by computing running averages and differences via scalar products with scaling signals and wavelets. For the Daubechies wavelet transforms, the scaling signals and wavelets have longer supports than Haar wavelet, i.e., they produce averages and differences using more values from the signal. This change, provides a big improvement in the capabilities of these new transforms.

Biorthogonal Wavelets: are preferable to use smooth mother wavelet for image analysis. Pair of scaling and wavelets functions used in Biorthogonal wavelets for analysis and synthesis. The analysis and synthesis wavelets can have different numbers of vanishing moments and regularity properties. Wavelet with the greater number of vanishing moments used for analysis resulting in a sparse representation [14], and the smoother wavelet used for reconstruction [15].

Symlets wavelets are nearly symmetrical wavelets Proposed by Daubechies as modifications to the Db family, apart from the symmetry, remaining other properties of Daubechies and Symlet families are similar [13, 16].

Coiflet wavelets: were derived from the Daubechies wavelet [17], to have scaling functions with vanishing moments. it uses six scaling and wavelet function coefficients, so increase in pixel averaging and differencing leads to a smoother wavelet and increased capabilities in several image-processing techniques (de-noising images, etc.). The filter has the same structure as both Haar and Daubechies. It has both averages and differences using the same format, only with six adjacent pixels.

The 5/3 and 9/7 lifting 2D Discrete Wavelet Transform: Lifting Scheme consists of three steps [18, 19]: Split, Predict and Update. Split image bit stream into odd and even elements. Data set predicted by approximating it to odd and even elements. The odd elements re replaced by the differences between the approximation and the actual data. While the even elements are left unchanged and become the input for the next step in the transform. Update the even elements with an average. These results in a smoother input for the next step of the wavelet transform. The odd elements represent an approximation of the original data set, which allows filters to be constructed. The original values of the odd elements have been overwritten by the difference between the odd element and its even "predictor". So in calculating an average the Update phase must operate on the differences that are stored in the odd elements [20-21].

SPIHT algorithm: The basic idea that the coefficients corresponding to the same spatial location in different sub bands in the pyramid structure display self-similarity characteristics [22]. SPIHT defines parent-children 
relationships between these self- similar sub bands to establish spatial orientation trees, see [21] for more information.

\section{Proposed Algorithm}

Medical images have special properties for compression than any other image types because you have not to degrade the image quality because the physicist decision about the treatment. So you need good compression ratio and in the same time you need a good quality image. To solve this problem, we introduce a compression technique based on combining wavelets with RG method. RG is used to separate the image into foreground and background based on image based on the pixel's intensities. The foreground regions (details) are compressed wavelets (as Daubechies, Biorthogonal, Coiflets, Symlets, Dmeyer, lifting 5/3 and 9/7). The output is leading to that the overall compression ratio gets better metrics PSNR, MSE, CR, and the transform time (T) than the existing wavelets methods. The vital steps of the proposed algorithm can be shown in the flowchart in Figure 1.

The algorithm steps can be described as follows:

1) Apply region growing $(R G)$ to segment the image.

2) Repeat Step1 until all pixels in image have been allocated to a suitable region according its density.

3) Apply wavelet methods on the regions of interest losslessly.

4) Apply the Spiht algorithm on the rest of the image.

5) End.

Pseudo code:

method grow (image, seed, neighborhood_size)

Initialize 'segmentation'.

Initialize 'checked'.

Initialize empty stack, 'needs_check'.

Set 'segmentation' and 'checked' at 'seed' to true.

Add neighbor coordinates of 'seed' to 'needs_check'. while 'needs_check' is not empty:

Pop 'point' from 'needs_check'.

Set 'checked' at 'point' to true.

if the average of 'image' over 'neighborhood_size' distance from 'point' $\geq$ the value of 'image' at 'point'(threshold)

then

Set 'segmentation' to true at 'point'.

Add neighbor coordinates of 'point' to 'needs_check'. end if end while

return 'segmentation'

end method grow

Step-1: Initialization

Result $n=\left[\log _{2} \max [|C(i, j)|\}\right]$;

Let $L S P=o$;

Let $L I P=(i, j) \in H$

Let $L I S=(i, j) \in H \forall D(i, j) \neq 0$ also entries(LIS $) \in A$

Step-2: Sorting Process

for $i=1$ to length of $(\operatorname{LlP}(i, j))$

$S_{n}(i, j)$,

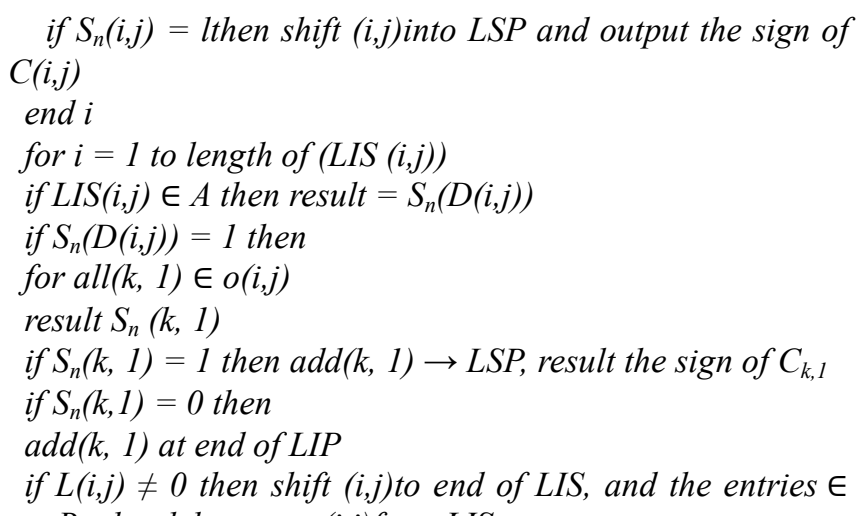
type $B$, else delete entry $(i, j)$ from LIS

for $i=1$ to length of $(\operatorname{LIS}(i, j))$

if $\operatorname{LIS}(i, j) \in B$ then result $=S_{n}(L(i, j))$

if $S_{n}(L(i, j))=1$ then

add each $h_{m}$ to the end of the LIS under A, else delete $(i, j)$ from $L S P$.

Step-3: Refinement Process

for $i=$ all $(i, j)$ in $L S P \forall i$ not appear in sorting process

$n^{\text {th }}$ important bit of $\left|C_{t J}\right|$

end $i$

Step-4: Quantization Pass

$n--/ /$ decrement $n$

goto Step -21

Stop

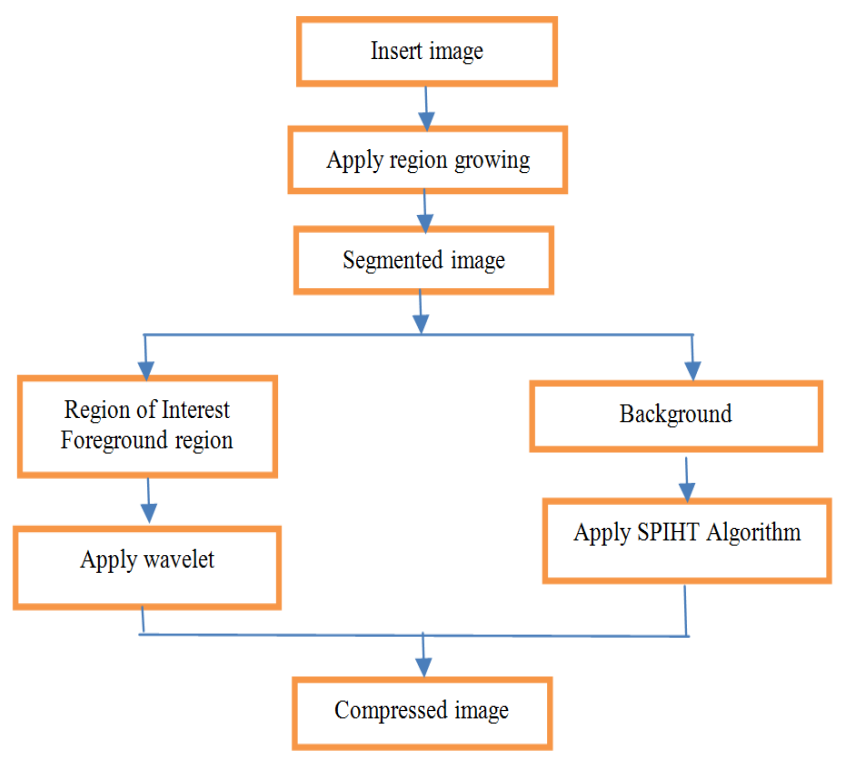

Figure 1. Flowchart for the proposed algorithm.

\section{Performance Evaluation}

Digital image compression techniques are normally analysed with objective fidelity measuring metrics like peak signal to noise ratio (PSNR), mean square error (MSE), and compression ratio (CR). These characteristics are used here to judge compression algorithms performance. 


\subsection{Mean Square Error (MSE)}

Refers to a sort of average or sum of squares of the error between two images. Root mean squared error or RMSE calculated by the square root of MSE. MSE for monochrome images is given by:

$$
M S E=\sqrt{\frac{\sum_{x=0}^{W-1} \sum_{y=0}^{H-1}\left[f(x, y)-f^{\prime}(x, y)\right]^{2}}{W H}}
$$

$f^{\prime}(x, y)$ is the pixel value of the decoded image while $\mathrm{f}(\mathrm{x}$, y) represent the pixel value of the original image,. Image compression systems are designed in order to minimize the MSE and maximize the PSNR.

\subsection{Peak Signal to Noise Ratio (PSNR)}

It is the ratio between signal variance and reconstruction error variance. PSNR is usually expressed in Decibel scale. The PSNR used as a measure of the reconstruction quality in image compression.

$$
\mathrm{PSNR}=10 * \log _{10} \frac{255^{2}}{\mathrm{MSE}}
$$

When the pixels are represented using 8 bits then 255 represent the maximum pixel value of the image, PSNR values can range between infinity for identical images, to 0 for images that have no commonality. PSNR is inversely proportional to MSE and CR that when PSNR decreases the $\mathrm{CR}$ increases, for the image.

\subsection{Compression Ratio (CR)}

Is defined as the ratio between the original image size and compressed image size.

$$
\mathrm{CR}=\frac{\text { original image }}{\text { compressed image }}
$$

\section{Experimental Results}

To prove the capability of the proposed algorithm, four different images structures from (X-Ray), Computed tomography (CT) and Magnetic resonance imaging (MRI) types as shown in Figure $2(\mathrm{a}, \mathrm{b}, \mathrm{c}, \mathrm{d})$ respectively were tested. Figure $2 \mathrm{a}$ shows the posterior cruciate ligament with dimensions $1280 \times 1280$. CT image as shown in Figure $2 \mathrm{~b}$ depicts the horizontal view for the Horizontal view for the human brain with dimensions $937 \times 937$. Figure 2 c shows $X$ ray image where the chest views with dimensions $1060 \times$ 1227. Figure $2 d$ shows MRI image of the circulatory system view with dimensions $1024 \times 610$.

We noted that combining region growing segmentation algorithm with a wavelet method gave higher CR while preserving the level for PSNR value in all image types.

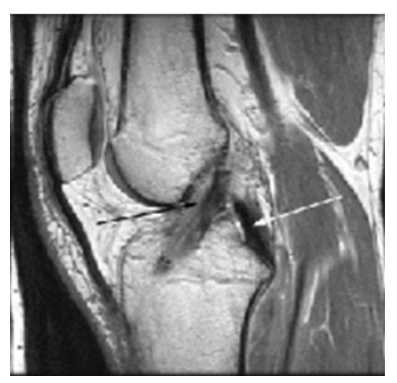

(a) Posterior cruciate ligament.

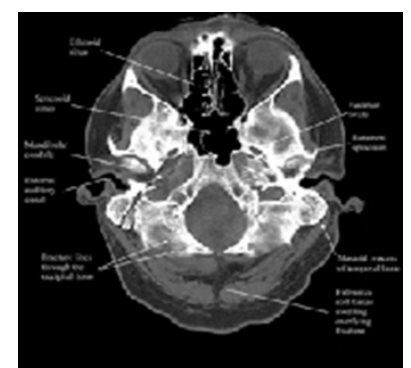

(b) Horizontal view for the human brain.

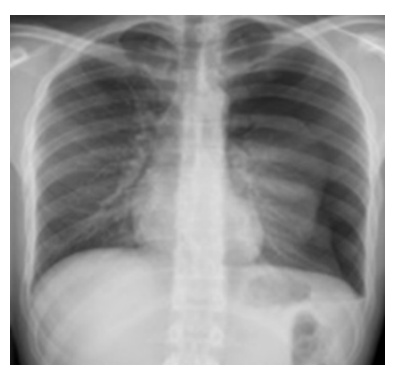

(c) Chest view.

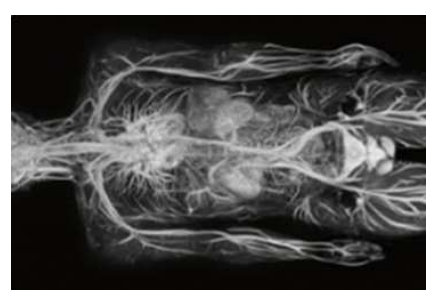

(d) Circulatory system.

Figure 2. Four image structures from X-Ray, Computed tomography CT and Magnetic resonance imaging MRI types.

This results are depicted in Table 1. The original images are first segmented, and then the interested part is coded by SPIHT wavelet as shown in the second column in Tables 2-5. The inverse transformation used to construct the final images (constructed image) as shown in the third column in Tables 2-5.

Metrics like PSNR, MSE, CR, and the transform time (Time) are used for each image to compare the results achieved by the classical wavelets like Daubechies, biorthogonal, Symlets, Coiflet, Dmeyer, lifting 5/3 and 9/7 methods and the proposed algorithm. A good behavior of the compression can be achieved for low value of MSE and according to the inverse relation between the MSE and PSNR, Logically, a high value of PSNR seems good for 
image compression; because it means that the ratio of signal to noise is high. So, if you find a compression scheme having a lower MSE and a high PSNR you can recognize that it is the best one. The lifting $5 / 3$ and $9 / 7$ gave best CR results between other wavelet methods for all test images. It is noticeable from Table 1 that the proposed algorithm achieved better CR more than three times the existing wavelets while preserving the same level of PSNR values. The results are promising when applying Lifting $9 / 7+$ SPIHT on the posterior cruciate ligament. The proposed method is achieved CR value (28.618) better than Lifting 9/7 (9.0528) by three times, while it saved almost the same level for the PSNR value. Similar for the CT Horizontal view for the human brain image, CR value equals 36.3285 for the proposed method and 10.3219 for the existing method. Testing the human chest X-Ray image and the circulatory system gave increasing in CR by three times than the existing wavelets. It is clear that the performance of the proposed method is depended on segmenting the image into foreground and background, i.e. good segmentation yields to increase in CR values without affecting the PSNR values. For that the proposed method gave better results than the existing wavelets for all test images as in Table 1. Although the time used for operating the proposed method is higher than the existing one, this is acceptable if the quality is needed.

Table 1. Results of CR, PSNR, MSE, Compression Time for the proposed and compared algorithms.

\begin{tabular}{|c|c|c|c|c|c|}
\hline \multirow{2}{*}{ Image } & \multirow{2}{*}{ Method } & \multicolumn{2}{|l|}{$\overline{C R}$} & \multicolumn{2}{|l|}{ PSNR } \\
\hline & & Wavelet & Proposed algorithm (RG + wavelets) & Wavelet & Proposed algorithm (RG + wavelets) \\
\hline \multirow{7}{*}{$\begin{array}{l}\text { the posterior } \\
\text { cruciate ligament } \\
\text { image }\end{array}$} & Lifting $9 / 7$ & 9.0528 & 28.618 & 33.584 & 32.214 \\
\hline & Lifting $5 / 3$ & 7.6401 & 28.5327 & 39.3425 & 35.215 \\
\hline & Coiflet & 1.7829 & 4.1254 & 40.0881 & 41.5624 \\
\hline & Daubechies & 2.0491 & 7.5286 & 30.1964 & 31.4857 \\
\hline & Biorthogonal & 2.0239 & 7.4185 & 32.0253 & 33.6514 \\
\hline & Symlets & 2.9024 & 8.6015 & 35.0815 & 36.2538 \\
\hline & Dmeyer & 1.1393 & 3.2084 & 30.2387 & 31.8524 \\
\hline \multirow{7}{*}{$\begin{array}{l}\text { Horizontal view } \\
\text { for the human } \\
\text { brain }\end{array}$} & Lifting 9/7 & 10.3219 & 36.3285 & 36.8349 & 34.8924 \\
\hline & Lifting $5 / 3$ & 8.9192 & 36.2506 & 38.0059 & 36.0927 \\
\hline & Coiflet & 2.1025 & 6.4256 & 36.6308 & 38.2451 \\
\hline & Daubechies & 2.3615 & 7.1025 & 25.4495 & 26.9494 \\
\hline & Biorthogonal & 2.4064 & 7.4523 & 28.2878 & 29.3451 \\
\hline & Symlets & 2.9051 & 7.6124 & 29.9912 & 30.6051 \\
\hline & Dmeyer & 1.3593 & 3.4521 & 25.9514 & 27.3458 \\
\hline \multirow{7}{*}{$\begin{array}{l}\text { the human chest } \\
\text { X-Ray image }\end{array}$} & Lifting $9 / 7$ & 10.4056 & 32.7352 & 40.9174 & 39.2951 \\
\hline & Lifting $5 / 3$ & 9.1576 & 32.3712 & 39.3946 & 37.8272 \\
\hline & Coiflet & 2.3429 & 6.8023 & 37.1228 & 38.6521 \\
\hline & Daubechies & 2.6775 & 7.6384 & 30.1408 & 31.7021 \\
\hline & Biorthogonal & 2.5292 & 7.6952 & 30.4901 & 31.9348 \\
\hline & Symlets & 2.8059 & 7.4215 & 32.1218 & 33.8307 \\
\hline & Dmeyer & 1.4911 & 3.9654 & 30.1177 & 31.6509 \\
\hline \multirow{7}{*}{$\begin{array}{l}\text { the circulatory } \\
\text { system }\end{array}$} & Lifting $9 / 7$ & 9.8894 & 31.6891 & 34.5759 & 32.9072 \\
\hline & Lifting $5 / 3$ & 8.1385 & 31.5228 & 36.3114 & 34.0052 \\
\hline & Coiflet & 1.9801 & 5.7521 & 35.5678 & 37.2671 \\
\hline & Daubechies & 2.2243 & 7.1658 & 30.6045 & 32.1039 \\
\hline & Biorthogonal & 2.3521 & 7.0254 & 30.8809 & 32.3094 \\
\hline & Symlets & 2.3521 & 6.8439 & 27.706 & 29.2537 \\
\hline & Dmeyer & 1.2881 & 3.4096 & 30.8763 & 32.5099 \\
\hline
\end{tabular}

Table 1. Continued.

\begin{tabular}{llllll}
\hline \multirow{2}{*}{ Image } & \multirow{2}{*}{ Method } & MSE & \multicolumn{3}{l}{ Time } \\
\cline { 2 - 6 } & Wavelet & Proposed algorithm (RG + wavelets) & Wavelet & Proposed algorithm (RG + wavelets) \\
\hline \multirow{4}{*}{ the posterior } & Lifting 9/7 & 0.9052 & 1.9573 & 3.5904 & 27.14573 \\
cruciate ligament & Lifting 5/3 & 0.7624 & 1.9569 & 3.0654 & 25.5596 \\
image & Coiflet & 0.6635 & 0.6124 & 4.0043 & 26.3284 \\
& Daubechies & 0.6803 & 0.6209 & 4.0581 & 26.9842 \\
& Biorthogonal & 0.4036 & 0.4092 & 4.5106 & 26.4689 \\
& Symlets & 0.4895 & 0.4631 & 3.9491 & 26.9242 \\
& Dmeyer & 0.6869 & 0.6671 & 8.7209 & 31.6524 \\
Horizontal view & Lifting 9/7 & 1.3582 & 1.5998 & 2.9548 & 18.5019 \\
for the human & Lifting 5/3 & 1.0372 & 1.5788 & 2.5604 & 20.8228 \\
\multicolumn{1}{c}{ brain } & Daiflet & 0.2993 & 0.2604 & 4.5709 & 27.6214 \\
& Biorthochies & 0.2280 & 0.2129 & 4.0529 & 27.2315 \\
& Symlets & 0.4383 & 0.3984 & 3.9271 & 27.5584 \\
& Dmeyer & 0.6489 & 0.6097 & 3.4985 & 26.1254 \\
\hline
\end{tabular}




\begin{tabular}{llllll}
\hline \multirow{2}{*}{ Image } & \multirow{2}{*}{ Method } & MSE & \multicolumn{3}{l}{ Time } \\
\cline { 2 - 6 } & Wavelet & Proposed algorithm (RG + wavelets) & Wavelet & Proposed algorithm (RG + wavelets) \\
\hline \multirow{4}{*}{ the human chest } & Lifting 9/7 & 0.5305 & 1.7201 & 2.6069 & 24.6234 \\
X-Ray image & Lifting 5/3 & 0.7533 & 1.6996 & 2.7988 & 25.4239 \\
& Coiflet & 0.5352 & 0.5195 & 3.9281 & 25.5911 \\
& Daubechies & 0.6716 & 0.6248 & 4.5241 & 26.0525 \\
& Biorthogonal & 0.7279 & 0.6873 & 2.9619 & 23.6557 \\
& Symlets & 0.6059 & 0.6022 & 3.2985 & 25.9228 \\
& Dmeyer & 0.6681 & 0.6409 & 6.6049 & 27.9587 \\
the circulatory & Lifting 9/7 & 1.2853 & 0.8567 & 2.4016 & 24.1211 \\
system & Lifting 5/3 & 1.5033 & 0.8176 & 2.5732 & 24.9614 \\
& Coiflet & 0.7343 & 0.6808 & 4.6809 & 26.2055 \\
& Daubechies & 0.7473 & 0.6985 & 4.6929 & 26.1941 \\
& Biorthogonal & 0.7964 & 0.7622 & 3.9497 & 25.7782 \\
& Symlets & 0.8834 & 0.7654 & 4.6591 & 26.1398 \\
& Dmeyer & 0.7956 & 0.7699 & 7.6586 & 28.4699 \\
\hline
\end{tabular}

Table 2. Results for the human chest X-Ray image.

\begin{tabular}{|c|c|c|c|}
\hline & $\overline{\text { Original image }}$ & Transformed image & Reconstructed imag \\
\hline Lifting 5 & & & \\
\hline Lifting 9/ & & & \\
\hline Coiflet & & & \\
\hline Daubechi & & & \\
\hline Biorthog & & & \\
\hline Symlets & & & \\
\hline Dmeyer & & & \\
\hline
\end{tabular}


Table 3. Results for the Circulatory system.

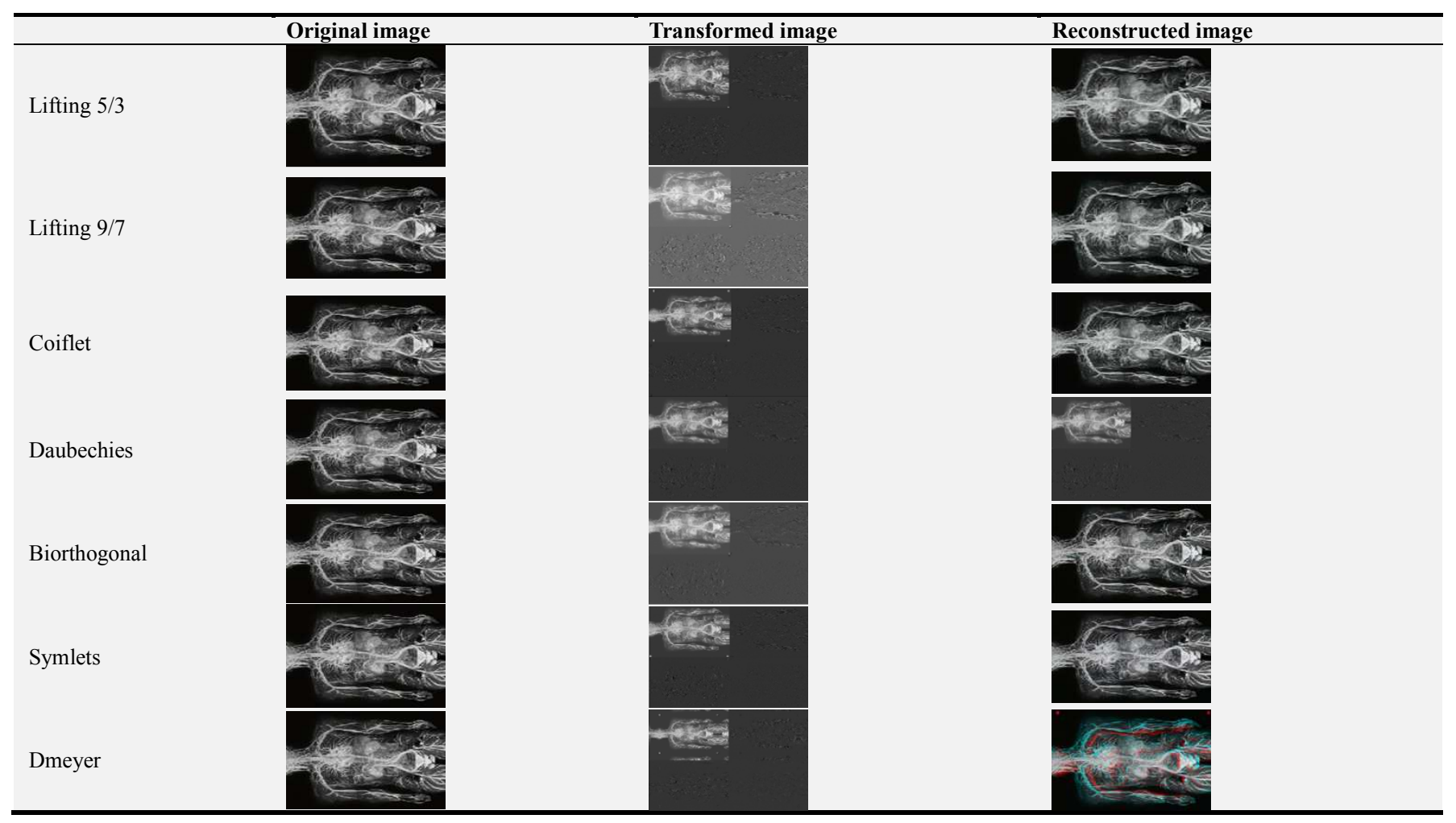

Table 4. Results for the Horizontal view for the human brain.

\begin{tabular}{|c|c|c|c|}
\hline & Original image & Transformed image & Reconstructed image \\
\hline \multicolumn{4}{|c|}{ Lifting $5 / 3$} \\
\hline \multicolumn{4}{|c|}{ Lifting $9 / 7$} \\
\hline \multicolumn{4}{|l|}{ Coiflet } \\
\hline \multicolumn{4}{|c|}{ Daubechies } \\
\hline \multicolumn{4}{|c|}{ Biorthogonal } \\
\hline Symlets & & & \\
\hline
\end{tabular}




\begin{tabular}{|c|c|c|c|}
\hline & Original image & Transformed image & Reconstructed image \\
\hline Dmeyer & & & \\
\hline
\end{tabular}

Table 5. Results for the posterior cruciate ligament image.

\begin{tabular}{|c|c|c|}
\hline & Transformed image & Reconstructed image \\
\hline Lifting 5 & & \\
\hline Lifting 9 & & \\
\hline Coiflet & & \\
\hline Daubech & & \\
\hline Biorthog & & \\
\hline Symlets & & \\
\hline Dmeyer & & \\
\hline
\end{tabular}

\section{Conclusion and Future Work}

In this paper, a compression technique based on combining RG and wavelets transform has been presented. The proposed algorithm started by segmenting the image into foreground and background regions to extract the important morphological details. Then, the algorithm was applied on the foreground region to compress the important details while background compressed using Spiht algorithm. The joining $\mathrm{RG}$ and wavelet leads to compress the image with greater CR while preserving same level of PSNR. The proposed algorithm has been tested with different four image structures from X-Ray, Computed tomography CT and magnetic resonance imaging MRI types to prove its efficiency. Experimental results collected of images for all wavelet types as shown in Table 1, where the best result for every parameter is bolded. This combination leads to increase CR values three times more than the existing wavelet methods. For example, the proposed method achieved $\mathrm{CR}$ value (28.618) better than Lifting 9/7 (9.0528) by three times, while it saved almost the same level for the PSNR value for the posterior cruciate ligament. Similar, the performance of the proposed method appears good rather than the existing one for all test images.

In the future, it could add the ability to enable manually 
selection of the regions needed to retain more details after compression to gain a clearer image to the lab specialist.

\section{References}

[1] A. Julius, "Medical Image Compression by Using Discrete Haar Wavelet Transform", International Journal of Science and Research (IJSR), 5 (9), 1196-1200 (2016).

[2] N. R. Rema, "Image Compression Using SPIHT with Modified Spatial Orientation Trees", International Conference on Information and Communication Technologies (ICICT 2014), Vol. 46, pp. 1732-1738, Procedia Computer Science (2015).

[3] N. Sriraam and R. Shyamsunder, "3-D medical image compression using 3-D wavelet coders", Elsevier on Digital Image Processing, 21 (1), 100-109 (2013).

[4] Y. Chen," Medical image compression using DCT-based sub band decomposition and modified SPIHT data organization", International journal of medical informatics, 76 (10), 717-725 (2007).

[5] A. Sharma and J. Bagga, "Lifting based 3D Discrete Wavelet Transform for Image Compression", International Journal for Research in Applied Science \& Engineering Technology (IJRASET), 6 (x), 69-73 (2018).

[6] G. K. Maye and T. Srinivasulu, "Image Compression Based on 3D DWT Coherent 13/7 Lifting Scheme Using VLSI Implementation for the Floating Point Multiplier Algorithm", International Journal of Pure and Applied Mathematics, 119 (18), 49-59 (2018).

[7] S. Sridhar and P. R. Kumar, "Wavelet Transform Techniques for Image Compression", I. J. Image, Graphics and Signal Processing, 2, 54-67 (2014).

[8] P. Vasanth, S. Rajan and L. Fred, "Efficient oppositional based optimal Harr wavelet for compound image compression using MHE”, Biomedical Research, 29 (10), 2169-2178 (2018).

[9] Basma A. Mohamed and Heba M. Afify, Mammogram Compression Techniques Using Haar Wavelet and Quad Tree Decomposition Based Image Enhancement, Biomedical Engineering: Applications, Basis and Communications, 29 (5), $1-7,2017$.

[10] E. A. Zanaty and W. M. Abdelhafiz, "A performance study of classical techniques for medical image segmentation”, Intl. J. Informatics and Medical Data Processing 1, 2 (2016).
[11] Z. M. Abood and I. H. AL-Qinani, "Semi-Automatic Seeded Region Growing for Object Extracted in MRI", International Journal of Scientific \& Engineering Research, 7 (2), 22295518 (2016).

[12] S. Meenalosini and J. Janet, "Segmentation of Cancer Cells in Mammogram Using Region Growing Method and Gabor Features", International Journal of Engineering Research and Applications, 2 (2), 1055-1062 (2012).

[13] D. Gupta and S. Choubey, "Discrete Wavelet Transform for Image Processing", International Journal of Emerging Technology and Advanced Engineering, 4 (3), 598-602 (2015).

[14] A. Graps, "An Introduction to Wavelets", IEEE Computational Science and Engineering, 2 (2), 1-18 (1995).

[15] A. Z. Averbuch, "Spline and Spline Wavelet Methods with Applications to Signal and Image Processing", Springer International Publishing, pp. 215-237, 2016.

[16] J. S. Walker, "A Primer on Wavelets and their Scientific Applications", Chapman \& Hall, 1998.

[17] A. Bakhshi and J. Singh, "Coiflet Wavelet Transform Image Compression Based On JPEG Images", International Journal of Advanced Research in Electrical, Electronics and Instrumentation Engineering, 5 (7), 6358-6363 (2016).

[18] B. E. Reddy and K. V. Narayana, "A lossless image compression using traditional and lifting based wavelets", International Journal of Image, Graphics and Signal Processing (SIPIJ), 3 (2), 213-222 (2012).

[19] J. Patel and K. Pathak, "Implementation of the 5/3 Lifting 2D Discrete Wavelet Transform", International Journal of Engineering Development and Research, 2 (3), 2953-2957 (2014).

[20] S. Zhang and G. Yang, "A Novel 9/7 Wavelet Filter Banks for Texture Image Coding", Signal\& Image Processing: An International Journal (SIPIJ), 3 (2), 7-14 (2012).

[21] R. C. Gonzalez, R. E. Woods, "Digital Image Processing", Prentice Hall, Upper Saddle River, NJ, 2002.

[22] S. Narasimhulu and T. Ramashri, "Gray-Scale Image Compression Using DWT-SPIHT Algorithm", International Journal of Engineering Research and Applications, 2 (4), 902905 (2012). 